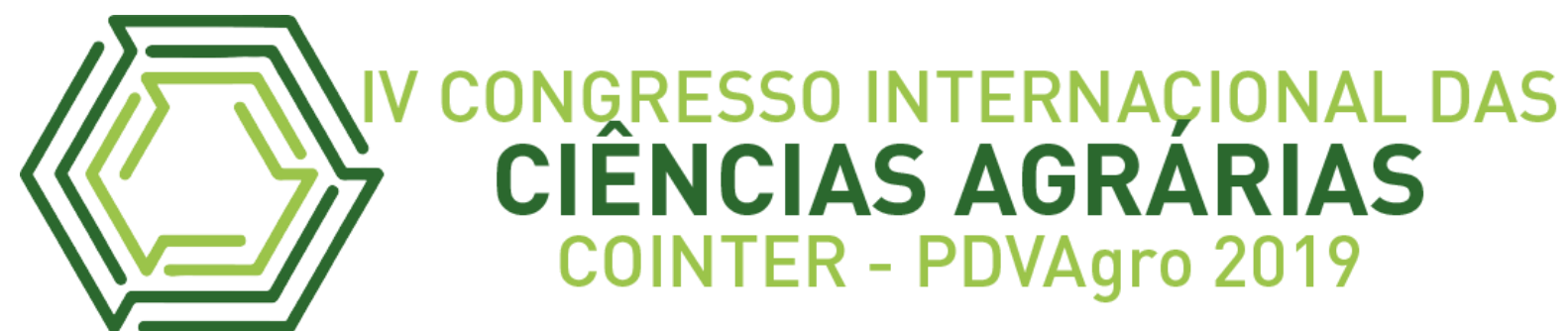

\title{
EFEITO DA APLICAÇÃO DE REVESTIMENTO A BASE DE GELATINA EM UVAS BENITAKA (VITIS VINIFERA) DURANTE O ARMAZENAMENTO A TEMPERATURA AMBIENTE
}

\section{EFECTO DE LA APLICACIÓN DEL RECUBRIMIENTO A BASE DE GELATINA EN UVAS DE BENITAKA (VITIS VINIFERA) DURANTE EL ALMACENAMIENTO A TEMPERATURA AMBIENTAL}

\section{EFFECT OF APPLICATION OF GELATINE BASED COATING ON BENITAKA GRAPES (VITIS VINIFERA) DURING STORAGE AT ENVIRONMENTAL TEMPERATURE}

Apresentação: Comunicação Oral

\author{
Larissa Mylena Mendes Dias ${ }^{1}$; Krause Gonçalves Silveira Albuquerque ${ }^{2}$; Karina \\ Barbosa dos Santos ${ }^{3}$; Adjair José da Silva ${ }^{4}$; Sônara de França Sousa ${ }^{5}$
}

DOI: https://doi.org/10.31692/2526-7701.IVCOINTERPDVAgro.2019.0103

\section{Resumo}

Diversos materiais de revestimentos podem ser utilizados para a conservação pós-colheita de frutos, e nesse contexto, a gelatina é uma proteína de grande produção no Brasil, de baixo custo e que forma filmes flexíveis e de funcionalidades interessantes. Objetivou-se com o presente trabalho estudar o efeito da aplicação de filmes comestíveis à base de gelatina em uvas cv. benitaka armazenadas em temperatura ambiente. As uvas foram revestidas com soluções a 1, 2 e 3\% de gelatina, armazenadas em estufa incubadora B.O.D. (Biochemical Oxygen Demand) a $24,3^{\circ} \mathrm{C}\left( \pm 5^{\circ} \mathrm{C}\right)$, por 8 dias, analisadas a cada 2 dias quanto aos seguintes parâmetros: perda de massa, luminosidade, intensidade $\mathrm{a}^{*}$, intensidade $\mathrm{b}^{*}$, atividade de água, sólidos solúveis totais, $\mathrm{pH}$ e acidez total titulável. O delineamento experimental utilizado foi inteiramente casualizado em esquema fatorial $5(0,2,4,6$ e 8 dias de armazenamento $) \times 4(0,1 \%, 2 \%$ e $3 \%$ concentrações) e a variável perda de massa foi analisada por curva de regressão polinomial de segunda ordem para a obtenção dos coeficientes de determinação $\left(\mathrm{R}^{2}\right)$. De acordo com os

\footnotetext{
${ }^{1}$ Graduanda em Engenharia de Alimentos, UFRPE-UAG, larissammdias0@gmail.com

${ }^{2}$ Graduando em Engenharia de Alimentos, UFRPE-UAG, albuquerque.k.g.s@gmail.com

${ }^{3}$ Graduanda em Engenharia de Alimentos, UFRPE-UAG, barbosa.s00@outlook.com

${ }^{4}$ Engenheiro Agrônomo, IFPE-CVSA, adjairsilva.agronomia.ifpe@gmail.com

${ }^{5} \mathrm{Dr}^{\mathrm{a}}$. em Engenharia de Processos, UFCG, sonara_franca@yahoo.com.br
} 
resultados obtidos, os revestimentos não se mostraram eficientes contra a perda de massa das amostras, onde os maiores valores foram observados para o tratamento a 3\%. As uvas apresentaram coloração escura com intensidade vermelho-azulada durante todo período de armazenamento. A atividade de água apresentou decréscimos, enquanto o teor de sólidos solúveis totais aumentou, ambos influenciados pela perda de água das amostras. Sobre o pH e acidez total titulável, as amostras foram caracterizadas como ácidas, tal característica pode ser creditada ao retardamento do amadurecimento.

Palavras-chave: perda de massa, pós-colheita, intensidade de cor

\section{Resumen}

Se pueden usar varios materiales de recubrimiento para la conservación de frutas después de la cosecha, y en este contexto, la gelatina es una proteína de gran producción en Brasil, de bajo costo y que forma películas flexibles y características interesantes. El objetivo de este trabajo fue estudiar el efecto de la aplicación de películas de gelatina comestible en uvas cv. benitaka almacenado a temperatura ambiente. Las uvas fueron recubiertas con soluciones de gelatina al 1,2 y $3 \%$, almacenadas en una cámara de $\mathrm{DBO}$ a $24.3^{\circ} \mathrm{C}\left( \pm 5^{\circ} \mathrm{C}\right)$, durante 8 días, analizadas cada 2 días para los siguientes parámetros: pérdida de peso, luminosidad. , intensidad a *, intensidad $\mathrm{b} *$, actividad del agua, sólidos solubles totales, $\mathrm{pH}$ y acidez titulable total. El diseño experimental fue completamente al azar en un esquema factorial $5(0,2,4,6$ y 8 días de almacenamiento) $\times 4$ (concentraciones de $0,1 \%, 2 \%$ y $3 \%$ ) y la pérdida de masa variable se analizó por curva. de regresión polinómica de segundo orden para obtener los coeficientes de determinación $\left(\mathrm{R}^{2}\right)$. Según los resultados obtenidos, los recubrimientos no fueron eficientes contra la pérdida de masa de las muestras, donde se observaron los valores más altos para el tratamiento del 3\%. Las uvas fueron de color oscuro con intensidad rojo azulada durante todo el período de almacenamiento. La actividad del agua disminuyó, mientras que el contenido total de sólidos solubles aumentó, ambos influenciados por la pérdida de agua de las muestras. Con respecto al $\mathrm{pH}$ y la acidez titulable total, las muestras se caracterizaron como ácidas, esta característica puede atribuirse a la maduración tardía.

Palabras Clave: pérdida de masa, poscosecha, intensidad de color

\section{Abstract}

Several coating materials can be used for postharvest preservation of fruits, and in this context, 
gelatin is a protein of great production in Brazil, low cost and forming flexible films and interesting features. The objective of this work was to study the effect of the application of edible gelatin films on grapes $\mathrm{cv}$. benitaka stored at room temperature. The grapes were coated with 1,2 and $3 \%$ gelatin solutions, stored in a BOD chamber at $24.3^{\circ} \mathrm{C}\left( \pm 5^{\circ} \mathrm{C}\right)$, for 8 days, analyzed every 2 days for the following parameters: weight loss, luminosity. , intensity a *, intensity $b^{*}$, water activity, total soluble solids, $\mathrm{pH}$ and total titratable acidity. The experimental design was completely randomized in a factorial scheme $5(0,2,4,6$ and 8 days of storage $) \times$ $4(0,1 \%, 2 \%$ and $3 \%$ concentrations $)$ and the variable mass loss was analyzed by curve. of second order polynomial regression to obtain the determination coefficients $\left(\mathrm{R}^{2}\right)$. According to the results obtained, the coatings were not efficient against the mass loss of the samples, where the highest values were observed for the $3 \%$ treatment. The grapes were dark colored with bluish-red intensity throughout the storage period. Water activity decreased, while the total soluble solids content increased, both influenced by the water loss of the samples. Regarding the $\mathrm{pH}$ and total titratable acidity, the samples were characterized as acidic, this characteristic can be credited to the delayed ripening.

Keywords: mass loss, postharvest, color intensity

\section{Introdução}

O consumo regular de frutas, legumes e verduras faz parte de qualquer estratégia de promoção da saúde devido às evidências acumuladas nas últimas décadas (TASSITANO; CABRAL; SILVA, 2014) a viticultura é um exemplo disso, no Brasil, a atividade ocupa uma área de aproximadamente 83.700 hectares, com uma produção anual variando entre 1.300 e 1.400 mil toneladas (CAMARGO; TONIETTO; HOFFMANN, 2011), além das uvas serem usadas para a fabricação do vinho, muitas delas são populares por serem consumidas in natura.

Apesar da diversidade e da disponibilidade, sua vida útil geralmente é bastante reduzida e as perdas pós-colheita se dão por fatores de origem mecânica, fisiológicas ou microbianas (CIA et al., 2010). Baseado na preocupação de que cada vez mais os consumidores almejam por produtos de melhor qualidade, os filmes comestíveis e/ou biodegradáveis são uma alternativa para melhoria da qualidade pós colheita de frutas e hortaliças, uma vez que eles podem trazer diversas vantagens, tais como a melhoria da aparência, propriedades antimicrobianas, não toxicidade, propriedades não-poluentes, e não podem promover alterações no gosto ou odor original e devem ser de baixo custo (ASSIS et al., 2009; ELSABEE e ABDOU, 
2013).

Os compostos mais utilizados na elaboração de revestimentos comestíveis são os polissacarídeos, os lipídios e as proteínas, ou a combinação destes compostos, o que permite utilizar vantajosamente as distintas características funcionais de cada classe. Entre as proteínas de revestimento, a gelatina já foi utilizada em diversas frutas, tais como, banana (SOUSA; FEITOSA; FIGUEIRÊDO, 2018), tomate (OLIVEIRA,2011), carambola (OLIVEIRA, 2015) e uvas crimson (FAKHOURI,et al, 2007), entretanto os resultados são dependentes da fruta e das variáveis tempo e temperatura de armazenamento. Diante do exposto, objetivou-se com o presente trabalho estudar o efeito da aplicação de filmes comestíveis à base de gelatina, em uvas cv. benitaka armazenadas em temperatura ambiente.

\section{Fundamentação teórica}

A gelatina é uma proteína de origem animal amplamente produzida no Brasil, a baixo custo e com propriedades funcionais adequadas para a fabricação de biofilmes (FAKHOURI, et al., 2007). Uma grande característica da gelatina é que ela tem capacidade de formar filmes flexíveis, pois é um hidrocolóide extremamente versátil, ela é atualmente o mais utilizado, pois possui propriedades funcionais interessante (MALI, et al, 2004). Do ponto de vista prático, as características mais marcantes da gelatina são a solubilidade em água e a capacidade de formação de gel termo-reversível.

Filmes à base de gelatina são visualmente transparentes, de fácil manuseio e possuem valores elevados de resistência à tração (DAVANÇO, 2006). Zocche (2010) avaliou a aplicabilidade de três tratamentos (fécula de mandioca 1\%, gelatina 5\% e controle sem nenhum tipo de revestimento) na conservação de acerolas e verificou que o tratamento com o revestimento de gelatina apresentou melhor resultado com relação às características sensoriais, sobretudo, à análise visual, observando que o revestimento de gelatina teve maior aceitação seguido pelo revestimento de fécula de mandioca, pois o primeiro realçou a aparência dos frutos.

Diante da necessidade de alternativas de conservação pós-colheita de frutos, é de fundamental importância que estudos dirigidos ao desenvolvimento e incentivo do uso de biofilmes e/ou revestimentos sejam pesquisados, visando assim contribuir para que esta prática seja coerente e acessível em larga escala no setor agrícola. 


\section{Metodologia}

As uvas cv. benitaka foram obtidas no comércio local da cidade de Garanhuns - PE, livres de danos mecânicos e fisiológicos. As frutas foram transportadas até o Laboratório de Análise de Alimentos da Unidade Acadêmica de Garanhuns da Universidade Federal Rural de Pernambuco, em seguidas foram selecionadas, padronizadas e submetidas a higienização e sanitização em água clorada $1 \mathrm{ml} \mathrm{L}^{-1}$ por 10 minutos, posteriormente foram secas em temperatura ambiente e colocadas em bandeja de poliestireno.

Para a elaboração das soluções de revestimento, pesou-se em balança analítica a gelatina incolor, diluindo-a em água destilada morna $\left( \pm 50^{\circ} \mathrm{C}\right)$ até a completa homogeneização. As concentrações utilizadas foram de 1,2 e $3 \%$ e uma controle ( $0 \%)$. Posteriormente, as uvas foram mergulhadas nesta solução por 2 minutos e deixadas para secar, em temperatura ambiente, para que se fosse formado a película de recobrimento nos frutos.

Depois de secas, as uvas foram armazenadas em câmaras B.O.D para a manutenção da temperatura $\left(24,3 \pm 5^{\circ} \mathrm{C}\right)$ e analisadas a cada 2 dias, em triplicata, durante 8 dias, de acordo com os seguintes parâmetros: perda de massa: obtida após pesagem individual das frutas em balança analítica, considerando a diferença do peso inicial e em cada avaliação; Cor: através de espectrofotômetro Mini Scan Hunter Lab XE Plus, onde foram determinados os valores de $\mathrm{L}^{*}$ luminosidades; $a^{*}$ transição da cor verde (-a*) para o vermelho $(*+a)$; e b* transição da cor azul $\left(-b^{*}\right)$ para a cor amarela $\left(+b^{*}\right)$, utilizando-se os parâmetros do sistema CIELab; acidez total titulável, de acordo com as normas Analíticas do Instituto Adolfo Lutz (IAL, 2008); pH, com um pHmetro previamente calibrado com soluções tampão 7,0 e 4,0; sólidos solúveis (SS): determinado por leitura em refratômetro portátil de bancada; atividade de água, por leitura direta em Aqualab (Decagon), a $25^{\circ} \mathrm{C}$.

O delineamento experimental utilizado foi inteiramente casualizado em esquema fatorial $5(0,2,4,6$ e 8 dias de armazenamento $) \times 4(0,1 \%, 2 \%$ e $3 \%$ concentrações $)$. A variável perda de massa foi analisada por curva de regressão. Os resultados obtidos das análises físicoquímicas foram submetidos à análise de variância pelo teste de Tukey a $5 \%$ e o Teste T, utilizando o Software de Assistência Estatística ASSISTAT versão 7.7 (SILVA e AZEVEDO, 2016). Para a análise da perda de massa, foi realizada a regressão polinomial de segunda ordem onde o ajuste do modelo aos dados experimentais deu-se de acordo com os valores do coeficiente de determinação $\left(\mathrm{R}^{2}\right)$ em que houve efeito significativo do tempo. 


\section{Resultados e Discussões}

$\mathrm{Na}$ figura 1 podemos observar a perda de massa durante todo o período de armazenamento, sendo a máxima de $50 \%$ para a amostra com $3 \%$ de gelatina ao $6^{\circ}$ dia, seguido pelos tratamentos a $2 \%, 1 \%$ e controle. A equação polinomial de segunda ordem apresentou bons ajustes, particularmente para a amostra a 2 e $3 \%$, podendo ser utilizada para representar de forma confiável os dados experimentais.

Os resultados obtidos não corresponderam ao expectável, já que vários autores referem que os revestimentos a base de gelatina proporcionam uma ação retardadora sobre a perda de água por parte dos frutos ao longo do seu período de conservação. Os resultados corroboram com Costa et al. (2017), os autores relataram que goiabas revestidas com gelatina durante 7 dias a temperatura ambiente apresentaram perda de massa de aproximadamente $6,8 \%$ no terceiro dia e 21,5\% no sétimo dia, valores que foram bastante próximos ao controle.

A perda de água, não resulta só em perdas de peso, mas também está associada a perdas de qualidade, causadas principalmente por degradação de aparência, alterações na qualidade nutricional e alterações na textura do fruto, uma vez que a água é responsável pela turgidez dos bagos (EMBUSCADO e HUBER, 2009;). Segundo Ngcobo et al. (2012) e Thompson (2015), com perdas de peso superiores a 5\% os bagos perdem o brilho e a turgidez, ficando macios e enrugados, o que leva à diminuição do seu valor comercial.

Oliveira et al. (2011) ao estudar a aplicação de biofilme de gelatina com concentração de $10 \%$ em tomates armazenadas em temperatura ambiente verificou o aumento na perda de água dos frutos revestidos em comparação ao tratamento controle, situação associada possivelmente ao caráter higroscópico do material utilizado para a produção do revestimento.

Wolf (2007) em seu estudo evidencia a capacidade higroscópica do filme de gelatina demonstrando que este pode alcançar valores próximos a $65 \%$ de umidade de equilíbrio em base seca potencializando a perda por vapor de água de sua superfície de contato, mencionando ainda que a livre permeação do vapor d'água proveniente do fruto para a atmosfera. O próprio filme perde seu conteúdo de água, em percentuais que podem chegar a $80 \%$ de sua massa inicial 


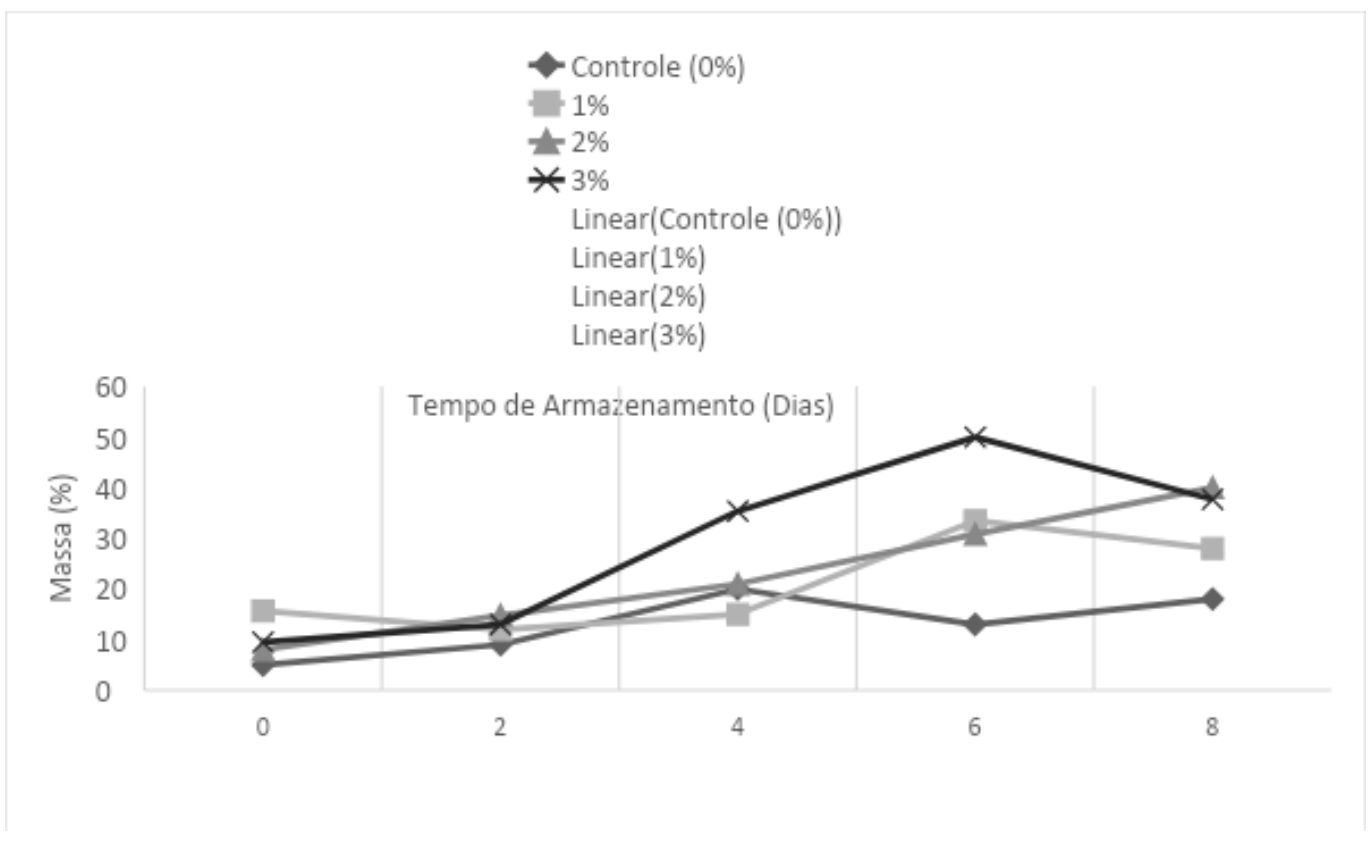

Figura 1- Perda de massa da uva cv. benitaka sobre diferentes revestimentos, armazenada em temperatura ambiente

Fonte: Própria (2019)

Na Tabela 1, tem-se a influência do tempo e da concentração do revestimento a base de gelatina sob a luminosidade das uvas cv. Benitaka. O parâmetro luminosidade estabelece uma escala cinza, com valores entre preto (0) e branco (100) (PATHARE et al., 2013), logo, as uvas apresentam-se escuras, com médias variando de 8,067 a 25,100. De acordo com o tempo, a luminosidade não apresentou uma tendência definida na amostra revestida a $1 \%$. A respeito da concentração, as médias não apresentaram diferenças significativas, exceto no $4^{\circ}$ dia de armazenamento, onde houve uma redução da luminosidade entre os tratamentos a 1 e $2 \%$.

Tabela 1- Valores médios da luminosidade em uvas cv. Benitaka sob diferentes concentrações de revestimentos a base de gelatina

\section{Luminosidade}

\begin{tabular}{cccccc}
\hline Concentração & \multicolumn{5}{c}{ Tempo de armazenamento (dias) } \\
\cline { 2 - 6 }$(\%)$ & 0 & 2 & 4 & 6 & 8 \\
\hline 0 & $14,067 \mathrm{aA}$ & $13,000 \mathrm{aA}$ & $19,967 \mathrm{abA}$ & $16,100 \mathrm{aA}$ & $16,133 \mathrm{aA}$ \\
1 & $8,067 \mathrm{aC}$ & $14,233 \mathrm{aBC}$ & $25,100 \mathrm{aA}$ & $20,833 \mathrm{aAB}$ & $12,200 \mathrm{aBC}$ \\
2 & $13,433 \mathrm{aA}$ & $20,367 \mathrm{aA}$ & $14,067 \mathrm{bA}$ & $14,000 \mathrm{aA}$ & $11,467 \mathrm{aA}$ \\
3 & $13,133 \mathrm{aA}$ & $12,100 \mathrm{aA}$ & $19,067 \mathrm{abA}$ & $14,700 \mathrm{aA}$ & $14,733 \mathrm{aA}$
\end{tabular}


Média 12,175

14,925

19,550

16,408

13,633

DMS para colunas $=9,188$. DMS para linhas $=9,794$. As médias seguidas pela mesma letras, maiúsculas nas linhas e minúsculas nas colunas, não diferem estatisticamente entre si ao nível de $5 \%$ de probabilidade. Fonte: Própria (2019)

Na Tabela 2, têm-se as médias da intensidade onde os valores positivos indicam a coloração vermelha das amostras. A análise estatística revelou que não houve variações significativas entre os tratamentos durante o armazenamento, onde também não é possível observar um comportamento definido para este parâmetro. A coloração das bagas é um dos atributos de qualidade mais atrativos para os consumidores, e de acordo com Mascarenhas et al. (2010), as uvas tintas, seguidas das rosadas, são preferidas em comparação às uvas de tons claros.

Tabela 2. Valores médios para a intensidade verde (-a*) e vermelho $\left(+\mathrm{a}^{*}\right)$ em uvas cv. Benitaka sob diferentes concentrações de revestimentos a base de gelatina

\begin{tabular}{cccccc}
\hline \multicolumn{5}{c}{ Parâmetro $\mathbf{\pm a}$} \\
\hline $\begin{array}{c}\text { Concentração } \\
(\%)\end{array}$ & 0 & \multicolumn{5}{c}{ Tempo de armazenamento (dias) } \\
\cline { 2 - 6 } & 3,400 & 2,667 & 8,600 & 4,500 & 3,900 \\
\hline 0 & 0,667 & 4.333 & 9,033 & 13,400 & 3,300 \\
1 & 4,500 & 8,200 & 5,867 & 9,700 & 1,133 \\
2 & 1,367 & 5,700 & 7,633 & 5,900 & 2,767 \\
3 & 2,483 & 5,225 & 7,783 & 8,375 & 2,775 \\
\hline
\end{tabular}

Não foi aplicado o teste de comparação de médias por que o $\mathrm{F}$ de interação não foi significativo. As médias seguidas pela mesma letra não diferem estatisticamente entre si. Foi aplicado o Teste t ao nível de 5\% de probabilidade. Fonte: Própria (2019)

Na Tabela 3 têm-se os valores médios para o parâmetro $\pm b^{*}$, onde valores negativos correspondem a coloração azul das amostras. De acordo com o ao tempo de armazenamento, observa-se que não houve uma redução significativa desta tonalidade para os tratamentos controle, a 1 e 3\%. Observando-se a concentração, todos os tratamentos não apresentaram variações na intensidade azul.

Tabela 3. Valores médios para a intensidade azul $\left(-b^{*}\right)$ e amarela $\left(+b^{*}\right)$ em uvas cv. Benitaka sob diferentes 
concentrações de revestimentos a base de gelatina

\section{Parâmetro $\pm \mathbf{b}$}

\begin{tabular}{cccccc}
\hline Concentração & \multicolumn{5}{c}{ Tempo de armazenamento (dias) } \\
\cline { 2 - 6 }$(\%)$ & 0 & 2 & 4 & 6 & 8 \\
\hline 0 & $-3,100 \mathrm{aA}$ & $-11,500 \mathrm{aA}$ & $-11,900 \mathrm{aA}$ & $-11,267 \mathrm{bA}$ & $-9,067 \mathrm{aA}$ \\
1 & $-9,000 \mathrm{aA}$ & $-6,500 \mathrm{aA}$ & $-21,300 \mathrm{aA}$ & $-11,433 \mathrm{bA}$ & $-6,933 \mathrm{aA}$ \\
2 & $-13,267 \mathrm{aB}$ & $-8,200 \mathrm{aB}$ & $-9,300 \mathrm{aB}$ & $-18,067 \mathrm{bA}$ & $-9,433 \mathrm{aB}$ \\
3 & $-10,767 \mathrm{aA}$ & $-6,533 \mathrm{aA}$ & $-13,133 \mathrm{aA}$ & $-10,767 \mathrm{bA}$ & $-10,267 \mathrm{aA}$ \\
Média & $-9,033$ & $-8,183$ & $-13,908$ & $-20,383$ & $-8,925$
\end{tabular}

DMS para colunas $=17.8125$ DMS para linhas $=18.9874$. As médias seguidas pela mesma letra não diferem estatisticamente entre si. Foi aplicado o Teste de Tukey ao nível de 5\% de probabilidade. Fonte: Própria (2019)

Na Tabela 4, têm-se os valores da atividade de água nas amostras. Observa-se que as médias diminuíram significativamente ao final do armazenamento ( $8^{\circ}$ dia $)$. Com relação à concentração, as médias quantificadas não apresentaram diferenças significativas, exceto no $6^{\circ}$ dias entre os tratamentos $2 \%$ e $3 \%$. A atividade de água está relativamente ligada à perda de massa das amostras, conforme observa-se na Figura 1. Müller et al. (2008) reportam que a permeabilidade ao vapor de água é influenciada muitas vezes pelas características intrínsecas do material de revestimento, pelo teor do plastificante e pelas condições de temperatura e umidade relativa do ambiente ao qual o filme é exposto.

Tabela 4. Valores médios da Atividade de água das em uvas cv. Benitaka sob diferentes concentrações de revestimentos a base de gelatina

\section{Atividade de água}

\begin{tabular}{cccccc}
\hline Concentração & \multicolumn{5}{c}{ Tempo de armazenamento (dias) } \\
\cline { 2 - 6 }$(\%)$ & 0 & 2 & 4 & 6 & 8 \\
\cline { 2 - 6 } 0 & $0,981 \mathrm{aA}$ & $0,979 \mathrm{aAB}$ & $0,965 \mathrm{aAB}$ & $0,979 \mathrm{aAB}$ & $0,961 \mathrm{aB}$ \\
1 & $0,964 \mathrm{aA}$ & $0,979 \mathrm{aA}$ & $0,975 \mathrm{aA}$ & $0,965 \mathrm{aA}$ & $0,961 \mathrm{aB}$ \\
2 & $0,976 \mathrm{aA}$ & $0,973 \mathrm{aA}$ & $0,978 \mathrm{aA}$ & $0,977 \mathrm{aA}$ & $0,953 \mathrm{aB}$ \\
3 & $0,971 \mathrm{aAB}$ & $0,978 \mathrm{aA}$ & $0,962 \mathrm{aABC}$ & $0,947 \mathrm{bC}$ & $0,957 \mathrm{aBC}$ \\
Média & 0,973 & 0,977 & 0,970 & 0,967 & 0,960
\end{tabular}

DMS para colunas $=0.0181 \quad$ DMS para linhas $=0.0193$ As médias seguidas pela mesma letra não diferem estatisticamente entre si. Foi aplicado o Teste de Tukey ao nível de 5\% de probabilidade. Fonte: Própria (2019) 
Na Tabela 5, têm-se os valores dos sólidos solúveis totais, estes representam todos os sólidos dissolvidos em água, como é o caso dos ácidos orgânicos, aminoácidos e pectinas solúveis, sendo que os valores da leitura realizada são referentes à soma de todos estes compostos (GULERIA, 2000). Os açúcares são o maior grupo de sólidos solúveis presentes nos frutos, pelo que a avaliação do conteúdo nestes sólidos pode ser usada para estimar o conteúdo em açúcares.

Verifica-se acréscimos significativos com relação ao tempo de armazenamento (controle e $2 \%$ ) e concentração (4 e 6 dias) do revestimento nas amostras. O aumento dos SST é geralmente relacionado à perda de água, as médias obtidas corroboram com Mascarenhas et al. (2013) ao avaliarem as características físico-químicas e sensoriais de uvas comerciais, encontrando valores entre 15,8 e $16,5^{\circ}$ Brix.

Tabela 5. Valores médios dos sólidos solúveis das em uvas cv. Benitaka sob diferentes concentrações de revestimentos a base de gelatina

\begin{tabular}{cccccc}
\hline \multicolumn{5}{c}{ Sólidos Solúveis Totais $\left({ }^{\circ}\right.$ Brix) } \\
\hline Concentração & \multicolumn{5}{c}{ Tempo de armazenamento (dias) } \\
\cline { 2 - 6 }$(\%)$ & 0 & 2 & 4 & 6 & 8 \\
\hline 0 & $13,233 \mathrm{cC}$ & $13,367 \mathrm{bC}$ & $15,133 \mathrm{bB}$ & $14,900 \mathrm{cB}$ & $24,233 \mathrm{aA}$ \\
1 & $17,333 \mathrm{aB}$ & $14,833 \mathrm{aC}$ & $14,233 \mathrm{cD}$ & $19,033 \mathrm{bA}$ & $17,100 \mathrm{~dB}$ \\
2 & $14,033 \mathrm{bC}$ & $12,267 \mathrm{cE}$ & $12,867 \mathrm{dD}$ & $18,900 \mathrm{bB}$ & $21,667 \mathrm{bA}$ \\
3 & $13,300 \mathrm{cD}$ & $12,367 \mathrm{cE}$ & $20,033 \mathrm{aB}$ & $29,767 \mathrm{aA}$ & $18,300 \mathrm{cC}$ \\
Média & 14,475 & 13,208 & 15,567 & 20,650 & 20,325 \\
\hline
\end{tabular}

DMS para colunas $=0.4590$ DMS para linhas $=0.4893$. As médias seguidas pela mesma letra não diferem estatisticamente entre si. Foi aplicado o Teste de Tukey ao nível de 5\% de probabilidade. Fonte: Própria (2019)

Com relação aos valores do pH das amostras (Tabela 6) observa-se que o tratamento a $2 \%$ não apresentou um comportamento definido com relação ao tempo de armazenamento. Analisando-se as colunas, verifica-se que as amostras não variaram significativamente ao 0,4 e 8 dias. Tal característica química deve ser observada na evolução da maturação, pois está 
relacionada com a qualidade das uvas. Resultado contrário pode ser detectados no trabalho de Oliveira et al. (2015) ao observarem as diferenças no $\mathrm{pH}$ de carambolas durante o armazenamento, independentemente do revestimento utilizado, com média variando de 1,63 a 12.

Tabela 6. Valores médios do pH das uvas cv. Benitaka sob diferentes concentrações de revestimentos a base de gelatina

\begin{tabular}{cccccc}
\hline \multicolumn{5}{c}{$\mathbf{p H}$} \\
\hline $\begin{array}{c}\text { Concentração } \\
(\%)\end{array}$ & 0 & 2 & 4 & 6 & 8 \\
\cline { 2 - 6 } & $3,900 \mathrm{aA}$ & $3,780 \mathrm{bA}$ & $3,870 \mathrm{aA}$ & $3,910 \mathrm{aA}$ & $3,920 \mathrm{aA}$ \\
0 & $3,840 \mathrm{aA}$ & $3,860 \mathrm{bA}$ & $3,850 \mathrm{aA}$ & $3,840 \mathrm{abA}$ & $3,870 \mathrm{aA}$ \\
1 & $3,880 \mathrm{aBC}$ & $5,810 \mathrm{aA}$ & $3,730 \mathrm{aBC}$ & $2,150 \mathrm{bC}$ & $4,150 \mathrm{aAB}$ \\
2 & $3,720 \mathrm{aA}$ & $3,680 \mathrm{bA}$ & $4,060 \mathrm{aA}$ & $4,230 \mathrm{aA}$ & $4,000 \mathrm{aA}$ \\
3 & 3,835 & 4,283 & 4,283 & 3,533 & 3,985
\end{tabular}

DMS para colunas $=1.6949$ DMS para linhas $=1.8067$. As médias seguidas pela mesma letra não diferem estatisticamente entre si. Foi aplicado o Teste de Tukey ao nível de 5\% de probabilidade. Fonte: Própria (2019)

Na Tabela 7 têm-se os valores médios da acidez total titulável, observa-se que as variações não foram significativas de acordo com o Teste t. Os resultado obtidos são superiores quando comparados com o de Rodrigues (2016) ao estudar uvas 'Crimson' conservada a $1^{\circ} \mathrm{C} \mathrm{e}$ UR 95\%, com a aplicação de revestimentos edíveis baseados em quitosano e Aloe vera, onde verificou um teor de ácido tartárico variando entre 0,35 a $0,41 \%$ ao longo dos 35 dias de armazenamento.

Tabela 7. valores médios da acidez titulável nas uvas cv. Benitaka sob diferentes concentrações de revestimentos a base de gelatina

Acidez Titulável (\% ácido tartárico)

\begin{tabular}{cccccc}
\hline \multirow{2}{*}{$\begin{array}{c}\text { Concentração } \\
(\%)\end{array}$} & \multicolumn{5}{c}{ Tempo de armazenamento (dias) } \\
\cline { 2 - 6 } & 0 & 2 & 4 & 6 & 8 \\
\hline 0 & 0,928 & 0,803 & 0,535 & 0,761 & 1,454 \\
1 & 0,688 & 0,841 & 0,440 & 0,933 & 0,876 \\
2 & 0,391 & 0,725 & 0,398 & 0,886 & 1,283
\end{tabular}


Média

$$
0,706
$$

0,805

0,458

0,738

Não foi aplicado o teste de comparação de médias por que o $\mathrm{F}$ de interação não foi significativo. As médias seguidas pela mesma letra não diferem estatisticamente entre si. Foi aplicado o Teste t ao nível de 5\% de probabilidade. Fonte: Própria (2019)

\section{Conclusões}

A partir do estudo realizado foi possível identificar que não houve variações decréscimos em relação a atividade de água do frutos, enquanto o teor de sólidos solúveis totais aumentou, ambos influenciados pela perda de água das amostras. No que diz respeito aos valores de $\mathrm{pH}$ e acidez, as amostras caracterizaram-se como ácidas, condição que pode ser creditada ao retardamento do amadurecimento. No tocante a cor dos frutos as uvas apresentam coloração escura com intensidade vermelho-azulada durante todo período de armazenamento. Entretanto, os revestimentos a base de gelatina não se mostram eficientes contra a perda de massa das amostras, isto pois, os frutos tratados com os revestimentos apresentaram maior perda de massa em comparação com o controle. Desta forma, faz-se necessário maiores estudos em relação às concentrações para uso da gelatina bem como a possibilidade de associação com outros compostos a fim de minimizar suas características higroscópicas.

\section{Referências}

ASSIS, S. A. Produção e caracterização de biofilme de quitosana com envoltório protetor em morangos, Recife. 2009. 88f. Tese de Doutorado (Nutrição). Universidade Federal de Pernambuco (UFRPE).

CAMARGO, U. A.; TONIETTO, J.; HOFFMANN, A. Progressos na viticultura brasileira. Revista Brasileira de Fruticultura, Jaboticabal - SP, volume Especial,p. 144-149, 2011.

CIA, P.; BENATO, E. A.; VALENTINI, S. R. T.; SANCHES, J.; PONZO, F. S.; FLÔRES, D.; TERRA, M. M. Atmosfera modificada e refrigeração para conservação pós colheita de uva 'Niagara Rosada'. Pesquisa Agropecuária Brasileira, Brasília, v.45, n.10, p.1058-1065, 2010.

COSTA, L. C.; SANTOS, L. R.; FRANÇA, R.; DAVINI, G. Aplicação de diferentes revestimentos comestíveis na conservação pós-colheita de goiabas (Psidium guajava L.). 
Brazilian Journal of Food Research, Campo Mourão, v. 8, n. 2, p. 16-31, 2017.

DAVANÇO, T. Desenvolvimento e caracterização de biofilmes à base de gelatina, triacetina, ácido esteárico ou ácido capróico e surfactantes. Campinas. 2006. 130 f. Dissertação (Mestrado em Alimentos e Nutrição) - Universidade Estadual de Campinas.

ELSABEE, M. Z.; ABDOU, E. S.; Chitosan based edible films and coatings: A review. Materials Science and Engineering C. Elsevier, v. 33, n. 4, p. 1819-18411, 2013.

EMBUSCADO, M.E., HUBER, K.C. Edible Films and Coatings for Food Applications, Springer. ed. Springer New York, New York, NY, 2009. doi:10.1007/978-0-387-92824-1

FAKHOURI, F. M.; FONTES, L. C. B.; GONÇALVES, P. V. M.; MILANEZ, C. R.; STEEL, C. J.; COLLARES-QUEIROZ, F. P. Filmes e coberturas comestíveis compostas à base de amidos nativos e gelatina na conservação e aceitação sensorial de uvas Crimson. Ciências e Tecnologia de Alimentos, Campinas, v.27, n.2, p. 369-375, 2007

GULERIA, S.P.S., 2000. Quality assurance for fruits, vegetables and their products, In: Verma, L.R., Joshi, V.K. (Eds.), Postharvest Technology of Fruits and Vegetables - Handling, Processing, Fermentation and Waste Management. Indus Publishing Company, New Delhi, India, pp. $201-234,2000$.

INSTITUTO ADOLFO LUTZ (IAL). Métodos Físico-químicos para análise de alimentos. 4.Ed. São Paulo: IAL,2008.

MALI, S.; GROSSMANN, M. V. E.; GARCÍA, M.; MARTINO, M.; ZARITZKY, N. E. Barrier, mechanical and optical properties of plasticized yam starch films. Carbohydrate Polymers, v. 56, n. 02, p. 129-135, jun. 2004.

MASCARENHAS, R. J.; SILVA, S. M.; LOPES, J. D.; LIMA, M. A. C. Avaliação sensorial de uvas de mesa produzidas no vale do são francisco e comercializadas em joão pessoa - PB. Revista Brasileira de Fruticultura, Jaboticabal - SP, v.32, n.4, p.993-1000, 2010 
MASCARENHAS, R. J.; GUERRA, N. B.; AQUINO, J. S.; LEÃO, P. C. S.; Qualidade sensorial e físico-química de uvas finas de mesa cultivadas no submédio São Francisco. Revista Brasileira de Fruticultura, Jaboticabal - SP, vol.35, n.2, pp.546-5542013.

MÜLLER, C. M. O.; YAMASHITA, F.; LAURINDO, J. B. Evaluation of effects of glycerol and sorbitol concentration and water activity on the water barrier properties of cassava starch films through a solubility approach. Carbohydrate Polymers, v.72, n.1, p.82- 87, 2008.

NGCOBO, M.E.K., DELELE, M.A., PATHARE, P.B., CHEN, L., OPARA, U.L., MEYER, C.J. Moisture loss characteristics of fresh table grapes packed in different film liners during $\begin{array}{lllll}\text { cold storage. } & \text { Biosyst. } & \text { Eng. }\end{array}$ DOI:10.1016/j.biosystemseng.2012.09.011

OLIVEIRA, T. A.; LEITE, R. H. L.; AROUCHA, E. M.M.; FERREIRA, R. M. A.Efeito do revestimento de tomate com biofilme na aparência e perda de massa durante o armazenamento. Revista Verde de Agroecologia e Desenvolvimento Sustentável, Mossoró, v. 6, n. 1, p. 230$234,2011$.

OLIVEIRA, T. A.; AROUCHA, E. M. M.; LEITE, R. H. L.; FERREIRA, R. M. A.; SANTOS, F. K. G. Conservação pós-colheita de carambola sob refrigeração com recobrimento de biofilme de gelatina e PVC. Revista Verde de Agrotecnologia, Pombal - PB, v. 10, n.4, p. 59 - 66, 2015

PATHARE, P. B.; OPARA, U. L.; AL-SAID, F. A-J. Colour measurement and analysis in fresh and processed foods. a review. Food and Bioprocess Technology, New York, v. 6, n. 1, p. 3660, 2013. DOI: https://doi.org/10.1007/s11947-012-0867-9

RODRIGUES, S. I. R. Conservação de uva de mesa 'Crimson' com recurso a revestimentos edíveis. Évora, 2016, 149f. Dissertação de Mestrado (Engenharia Agronômica). Universidade de Évora. 2016

SOUSA, S. F.; FEITOSA, R. M.; FIGUEIRÊDO, R. M. F. Aplicação de diferentes revestimentos na conservação pós-colheita da banana cv. Prata. Nativa, v. 6, n. 6, p. 563-568, 
nov./dez. 2018.

SILVA, F.A. S.; AZEVEDO, C. A. V. The Assistat Software Version 7.7 and its use in the analysis of experimental data. African Journal Agricultural Research, v.11, n.39, p.37333740, 2016.

TASSITANO, R. M.; CABRAL, P. C.; SILVA, G. A. P. Validação de escalas psicossociais para mudança do consumo de frutas, legumes e verduras. Caderno de Saúde Pública [online], v.30, n.2, p.272-282, 2014.

THOMPSON, A.K., 2015. Specific recommendation for fruit - Grapes, In: Fruit and Vegetables - Harvesting, Handling and Storage (Volume 1 - Introduction and Fruit). Wiley Blackwell, USA.

WOLF, K. L. Propriedades físico-químicas e mecânicas de biofilmes elaborados a partir de fibra e pó de colágeno,2007, 103p. Dissertação de Mestrado (Engenharia e Ciência de Alimentos), Universidade Estadual Paulista. 2007

ZOCCHE, L. Avaliação da eficiência, aceitação visual e sensorial de acerolas tratadas com biofi Imes comestíveis. Medianeira - PR. 2010. 22p. Trabalho de Conclusão de Curso, Universidade Tecnológica Federal do Paraná 